\title{
Absceso cerebral por Eikenella corrodens en un paciente pediátrico inmunocompetente
}

\author{
Gabriela E. Valdés-de la Torre* y María E. Martínez-Bustamante \\ Centro Médico Nacional 20 de Noviembre, Instituto de Seguridad y Servicios Sociales de los Trabajadores del Estado, Ciudad de México, México
}

\section{Resumen}

Introducción: Los abscesos cerebrales son una urgencia neurológica grave con alto riesgo de déficit neurológico permanente. Son patologías raras en la edad pediátrica, con una incidencia anual de 0.5 por 100,000 niños. Se han realizado pocos estudios de abscesos cerebrales de origen odontogénico y la mayoría de los pacientes son adultos con patología dental de base. Eikenella corrodens es un cocobacilo gramnegativo anaerobio facultativo de crecimiento fastidioso, que forma parte de la biota de la cavidad oral, pero es un patógeno frecuente en infecciones de cabeza y cuello, así como en infecciones dentales. Caso clínico: Paciente de sexo masculino de 16 años, previamente sano, que presentó cefalea de 2 meses de evolución acompañada de náuseas y vómitos. Antecedente de extracción del cuarto molar superior derecho 4 meses antes, sin uso de profilaxis antibiótica. La resonancia magnética de cráneo mostró una lesión compatible con absceso cerebral. Se realizó drenaje por punción guiada por estereotaxia, del que se aisló E. corrodens. El paciente completó un tratamiento con ceftriaxona y metronidazol por vía intravenosa durante 4 semanas y ampicilina-sulbactam por vía oral por 2 semanas más. Conclusiones: Los abscesos cerebrales de origen odontogénico causados por E. corrodens en la edad pediátrica son muy raros. Actualmente, el uso de antibióticos profilácticos en procedimientos dentales es controversial, ya que se ha documentado el aumento de la resistencia microbiana por su uso indiscriminado. La optimización de los estudios diagnósticos y el tratamiento multidisciplinario han mejorado el pronóstico de los pacientes con absceso cerebral.

Palabras clave: Absceso cerebral. Eikenella corrodens. Niños. Profilaxis antibiótica.

\section{Cerebral abscess due to Eikenella corrodens in an immunocompetent pediatric patient}

\section{Abstract}

Background: Brain abscesses are a serious neurological emergency with a high risk of permanent neurological deficit. The pathology is a rare in the pediatric age: the annual incidence is 0.5 per 100,000 children. There are a few studies of brain abscesses of oral origin mostly in adult patients with an underlying dental pathology. Eikenella corrodens, a slow-growing, Gram negative, facultatively anaerobic rod-bacillus, is part of the oral cavity biota, and common as a pathogen in head, neck, and dental infections. Case report: A previously healthy 16-year-old male presented a headache of 2 months of evolution accompanied by nausea and vomiting. Four months earlier, the fourth upper right molar was extracted, with no antibiotic prophylactic treatment. Magnetic resonance imaging of the skull showed a lesion compatible with brain abscess. Stereota$x y$-guided puncture drainage was performed, isolating E. corrodens. The treatment was with ceftriaxone and metronidazole

\section{Correspondencia:}

*Gabriela E. Valdés-de la Torre

E-mail: stephyvaldes@gmail.com
Fecha de recepción: 27-02-2020

Fecha de aceptación: 09-05-2020

DOI: 10.24875/BMHIM.20000049
Disponible en internet: 20-11-2020

Bol Med Hosp Infant Mex. 2021;78(2):136-142

www.bmhim.com

1665-1146/C 2020 Hospital Infantil de México Federico Gómez. Publicado por Permanyer. Este es un artículo open access bajo la licencia CC BY-NC-ND (http://creativecommons.org/licenses/by-nc-nd/4.0/). 
intravenously for four weeks and ampicillin sulbactam orally for two more weeks. Conclusions: Brain abscesses of odontogenic origin by E. corrodens in the pediatric age are very rare. Currently, the use of prophylactic antibiotics in dental procedures is controversial because the indiscriminate use increases antimicrobial resistance. The optimization of diagnostic studies and multidisciplinary treatment has improved the prognosis of patients with brain abscesses.

Key words: Brain abscesses. Eikenella corrodens. Children. Prophylactic antibiotics.

\section{Introducción}

Los abscesos cerebrales son una urgencia neurológica grave por el alto riesgo de causar déficit neurológico permanente ${ }^{1}$. A pesar del avance en su diagnóstico y tratamiento, las tasas de morbimortalidad aún son significativas ${ }^{2}$. La incidencia anual en la población general es de 0.3-1.3 casos por 100,000 habitantes $^{3}$. Esta patología es rara en la edad pediátrica: solo el $25 \%$ del total de los abscesos cerebrales se presentan en niños ${ }^{2,4}$. En una serie de casos de abscesos cerebrales en pacientes pediátricos se estimó una incidencia anual de 0.5 por 100,000 niños $^{3}$. La incidencia más frecuente es entre los 4 y los 7 años, y la localización varía según la edad: en lactantes y preescolares, son más comunes los abscesos cerebelares, y en los escolares y adolescentes, en la región temporal, con 1.5 a 3.1 de predilección por el sexo masculino ${ }^{4}$.

La incidencia pediátrica ha disminuido a lo largo de los años por una mejoría en el tratamiento de las infecciones sinusales y óticas. Los estudios reportan una incidencia del $15-30 \%$ en pacientes menores de 15 años $^{5}$. El $80 \%$ de los abscesos cerebrales presentan un factor predisponente y el resto son idiopáticos. Dentro de los factores predisponentes, el $30-50 \%$ se deben a un foco infeccioso de contigüidad (otitis media, mastoiditis, sinusitis, celulitis orbitaria), el $30 \%$ se asocian a bacteriemias (cardiopatía congénita, infección pulmonar e infección odontogénica), el 10\% son por inoculación directa y el $10 \%$ ocurren en individuos inmunocomprometidos $^{3,6-8}$. Los factores predisponentes varían entre niños y adultos, y los principales son las malformaciones cardiacas congénitas, el tracto dermosinusal congénito y los cuerpos extraños en los pulmones².

Existen pocos estudios en la literatura sobre abscesos cerebrales de origen odontogénico en niños. Se realizó una búsqueda de reportes de estos casos en la base de datos PubMed y se incluyeron 55 artículos con pacientes entre 3 y 70 años de edad. Sin embargo, estos reportes no mencionan cuántos pacientes pediátricos fueron estudiados.

La diseminación de bacterias de la cavidad oral se puede presentar por múltiples condiciones dentales, como gingivitis y periodontitis, o por procedimientos como extracciones dentales, endodoncia y cirugía oral. El cepillado dental por sí solo puede inducir bacteriemias transitorias en el $38.5 \%$ de los casos. Actualmente no se recomienda el uso sistemático de antibióticos profilácticos en procedimientos dentales para la prevención de abscesos cerebrales, dada la baja incidencia de esta complicación y el riesgo potencial de resistencia microbiana ${ }^{6}$.

Eikenella corrodens es un cocobacilo gramnegativo, anaerobio facultativo de crecimiento fastidioso, cuyo crecimiento se ve favorecido en presencia de dióxido de carbono al $5 \%$ y $10 \%$. Este microorganismo es oxidasa positivo, no fermentador de glucosa, y urea e indol negativo ${ }^{9}$. Forma parte de la biota de la cavidad oral, las vías respiratorias altas y la mucosa intestinal de los seres humanos ${ }^{4,9}$. Es miembro de la familia HACEK (Haemophilus, Aggregatibacter, Cardiobacterium, Eikenella y Kingella), cuyos miembros comparten un crecimiento lento y requerimiento de dióxido de carbono. Es frecuente como patógeno en infecciones de cabeza y cuello, infecciones dentales, heridas contaminadas por secreciones orales, infecciones pleuropulmonares, del sistema nervioso central y abdominales, osteomielitis, bacteriemias y endocarditis ${ }^{10}$. Las infecciones por Eikenella spp. en pacientes pediátricos son poco frecuentes y existen pocos casos reportados.

En un estudio realizado en el Rainbow Babies and Children's Hospital, de Ohio, se revisaron los casos de infecciones por Eikenella en niños y adolescentes en un periodo de 5 años (1994-1999), y se analizaron también datos obtenidos en la literatura. Se diagnosticaron 54 casos de infección por Eikenella: 13 casos del hospital y 41 casos de la literatura. La presentación clínica más común fue en cabeza y cuello, y la mayoría tenían comorbilidad infecciosa otorrinolaringológica ${ }^{10}$.

En este reporte se presenta el caso de un adolescente inmunocompetente con un absceso cerebral por E. corrodens de probable origen odontogénico, sin factores de riesgo asociados.

\section{Caso clínico}

Paciente de sexo masculino de 16 años, previamente sano, que ingresó al hospital por presentar cefalea de 
2 meses de evolución acompañada de náusea y vómito. Como antecedente de importancia, acudió con maxilofacial, quien realizó extracción del cuarto molar superior derecho, sin recibir profilaxis antimicrobiana. Esquema de vacunación incompleto, con la última dosis de neumococo e influenza estacional pendiente.

Cuatro meses después, el paciente inició con cefalea hemicraneana derecha, intermitente, intensidad de dolor 5/10, exacerbada con los cambios de posición. Presentó mejoría con la administración de medicamentos antiinflamatorios no esteroideos (intensidad de dolor $3 / 10$ ), sin otra sintomatología asociada.

Un mes después continuó con cefalea de las mismas características, agregándose anhedonia, y al siguiente mes incrementó la intensidad de la cefalea (8/10), acompañada de dolor retroocular, náusea y vómito, por lo que se decidió su ingreso hospitalario para valoración y tratamiento. Ingresó por datos sugestivos de hipertensión endocraneana. A la exploración física, se encontró con signos vitales dentro de los parámetros para su edad, consciente, orientado, cooperador, Glasgow 15, pupila izquierda hiporreactiva, oídos sin alteraciones, cavidad bucal con amígdalas hipertróficas III/IV y encriptadas, halitosis, neurológicamente anhedónico, razonamiento y juicio conservados. No le fue posible realizar síntesis ni abstracción, y cálculo con dificultad. Pares craneales: fondo de ojo normal, reflejo fotomotor y consensual presente bilateral, movimientos oculares presentes, sensibilidad en la cara conservada, con simetría facial, reflejo nauseoso presente, úvula y lengua central, resto de los pares sin alteraciones. Tono global normal, trofismo conservado, hiperreflexia en las cuatro extremidades. Marcha independiente, realizó puntas y talones, y tándem con dificultad, sensibilidad conservada, adecuada metría y disdiadococinecia. Los resultados de sus análisis de laboratorio al ingreso fueron los siguientes: hemoglobina, $15.3 \mathrm{~g} / \mathrm{dl}$; hematocrito, $45 \%$; leucocitos, $10,200 / \mathrm{mm}^{3}$; neutrófilos, $80 \%$; linfocitos, $15 \%$, y plaquetas, $301,000 / \mathrm{mm}^{3}$. Química sanguínea y electrolitos séricos dentro de parámetros normales.

Se solicitó tomografía computarizada (TC) simple de cráneo de urgencia, en la que se observó una lesión esférica en el hemisferio derecho e hipodensidad difusa periférica (Figura 1). Se solicitó resonancia magnética (RM) de cráneo con gadolinio para mayor definición de las características de la lesión, y se reportó lesión compatible con absceso cerebral localizado en los núcleos de la base derecha, con reforzamiento anular y edema perilesional que sugieren fase de cápsula tardía de $5.7 \times 3.6 \mathrm{~cm}$ (Figura 2). El paciente fue

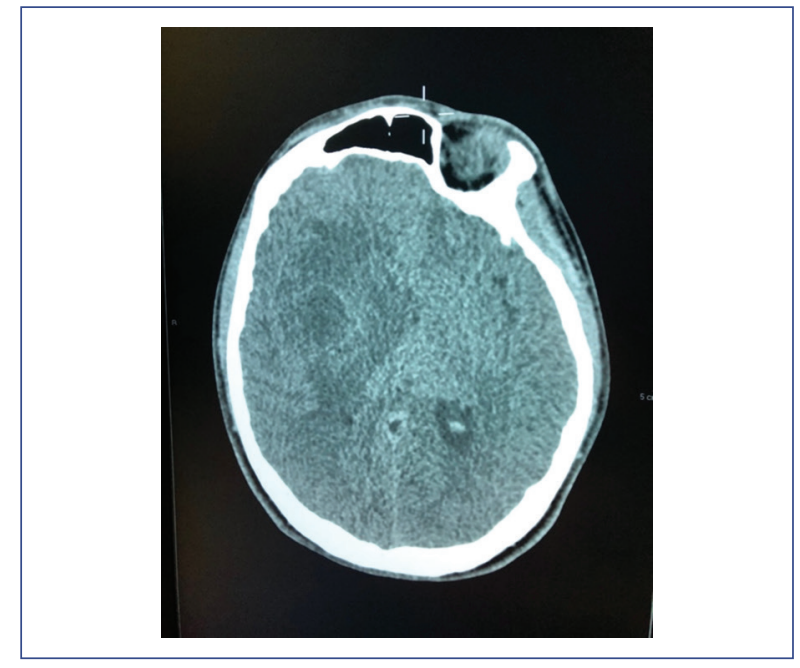

Figura 1. Tomografía computarizada de cráneo simple con lesión hipodensa en la región temporal derecha.

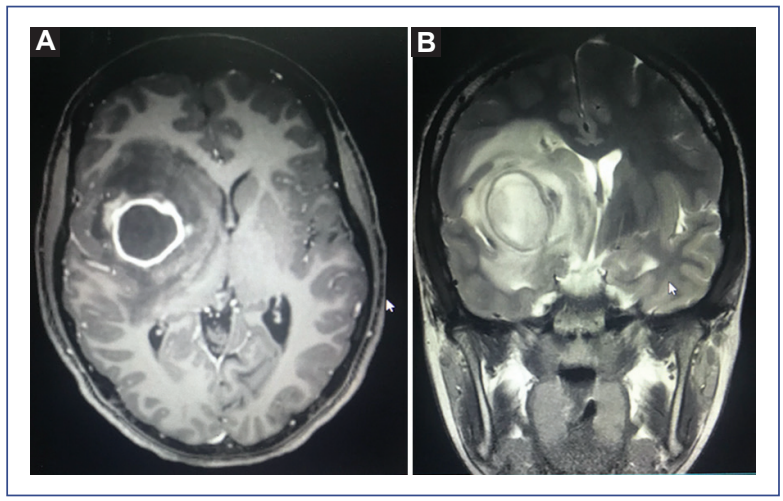

Figura 2. A: resonancia magnética de cráneo en T1 con medio de contraste. Se observa lesión compatible con absceso cerebral con reforzamiento anular. B: resonancia magnética en T2.

valorado por el servicio de infectología y se sospechó un absceso cerebral. Inició con tratamiento empírico por vía intravenosa de ceftriaxona $(100 \mathrm{mg} / \mathrm{kg}$ al día, cada $12 \mathrm{~h}$ ) y metronidazol (30 mg/kg al día, cada 8 h). Como parte del manejo multidisciplinario, el servicio de neurocirugía realizó el drenaje quirúrgico por punción guiada por estereotaxia, drenando aproximadamente $10 \mathrm{ml}$ de material purulento, que se envió a cultivo y patología. A las 48 horas del cultivo en agar chocolate incubado a $37^{\circ} \mathrm{C}$ se observaron colonias puntiformes, circulares, de color gris (Figura 3). La tinción de Gram reportó bacilos gramnegativos, y las pruebas bioquímicas los identificaron como oxidasa positivos, catalasa negativos y urea e indol negativos, por lo que se reportó E. corrodens. 


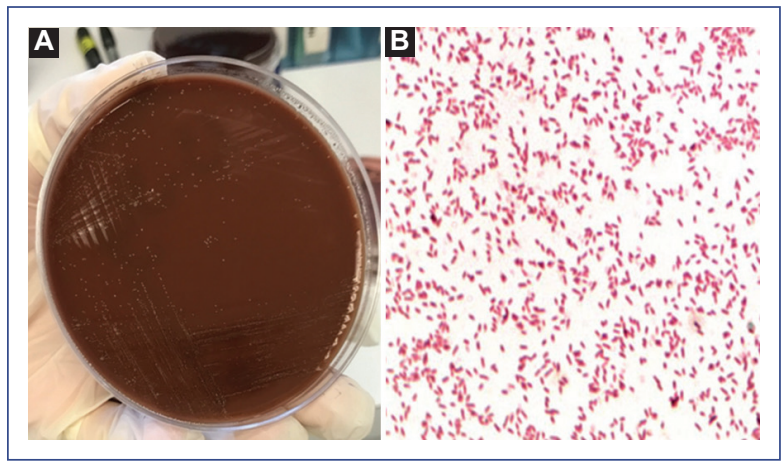

Figura 3. A: colonias puntiformes en agar chocolate. B: tinción de Gram: bacilos gramnegativos.

Dentro del abordaje se investigaron los probables orígenes de la infección, descartando un foco de contigüidad (otitis media, sinusitis). Se realizó un ecocardiograma, en el que no se observaron cardiopatías ni datos de endocarditis. Por el antecedente de extracción dental y por el aislamiento de un patógeno que forma parte de la biota de la cavidad oral, se sospechó un probable origen odontogénico.

Se continuó con el tratamiento previamente establecido por la alta posibilidad de una etiología polimicrobiana y se completaron 4 semanas de tratamiento intravenoso con ceftriaxona y metronidazol, y 2 semanas más con ampicilina-sulbactam por vía oral. A las 2 semanas de iniciado el tratamiento antibiótico y el drenaje, se realizó una nueva RM de cráneo en la que se observó una disminución importante del tamaño de la lesión (Figura 4). Dos semanas posteriores al egreso, en consulta de seguimiento, el paciente presentó una adecuada evolución clínica, sin cambios en el estado neurológico. La RM no mostró ninguna lesión aparente. Se mantuvo en observación por 1 año, con citas cada 3 meses y una RM más de control a los 6 meses, sin datos de recurrencia. El paciente no presentó secuelas neurológicas.

\section{Discusión}

A pesar de los avances en el diagnóstico y tratamiento, los abscesos cerebrales continúan siendo un reto para los médicos, ya que las manifestaciones clínicas y radiológicas de esta patología son inespecíficas $^{3}$. En una revisión sistemática de 9,699 casos de absceso cerebral (1935-2012) se observó que las formas de adquisición más comunes fueron por un foco de contigüidad (en el $40-50 \%$ de los casos: otitis/mastoiditis $33 \%$, sinusitis $10 \%$ ) y por vía hematógena (en

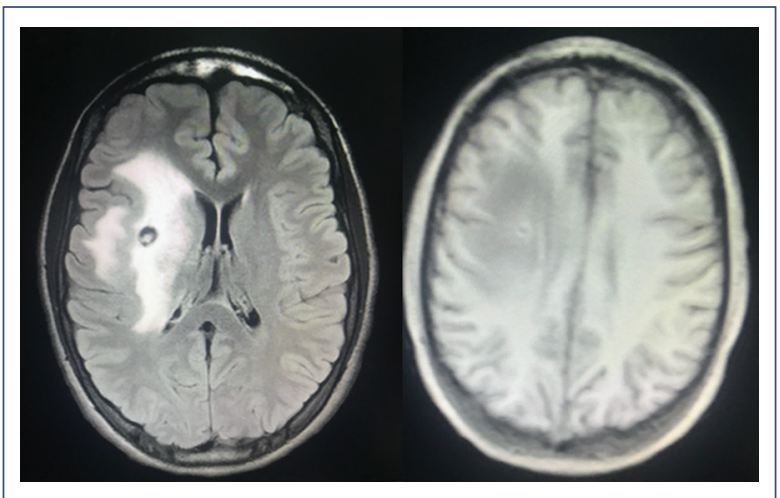

Figura 4. Resonancia magnética de cráneo de control a las 2 semanas postratamiento.

el $33 \%$ de los casos: endocarditis $13 \%$, infección pulmonar $8 \%$, infección dental $5 \%)^{6,11-13}$.

En este caso se sospechó un origen odontogénico por el procedimiento dental previo a la manifestación de los síntomas y por el aislamiento de una bacteria que forma parte de la biota de la cavidad oral. Ewald, et al..$^{14}$ propusieron criterios diagnósticos para abscesos cerebrales de origen odontogénico: que no presenten otra fuente identificada de bacteriemia asociada ${ }^{2}$ causada por microorganismos comúnmente encontrados en la cavidad oral ${ }^{3}$ ni síntomas clínicos o radiográficos de una patología dental o antecedente de un procedimiento dental 1 a 4 semanas antes del inicio de la sintomatología ${ }^{6}$.

La cavidad oral presenta abundante microbiota: alrededor de 1,200 tipos de bacterias. La vía de entrada por un foco odontogénico puede ser hematógena, por drenaje venoso directo al seno cavernoso, por extensión directa al introducir objetos extraños o por drenaje linfático ${ }^{8}$. Múltiples condiciones y procedimientos dentales, como gingivitis, periodontitis, caries, extracción dental, endodoncia y cirugía oral, pueden ocasionar abscesos cerebrales, la mayoría polimicrobianos ${ }^{8}$. Entre las bacterias que se han aislado con mayor frecuencia se encuentran Streptococcus viridans, Actinomyces, Peptostreptococcus, Prevotella, Fusobacterium, Aggregatibacter actinomycetemcomitans y E. corrodens ${ }^{1,6,11}$.

Debido a la baja incidencia de esta patología, existen pocas publicaciones sobre infecciones intracraneales de origen odontogénico. En una revisión sistemática de la literatura sobre infecciones intracraneales de origen odontogénico se incluyeron 55 artículos, con un total de 60 pacientes de 3-70 años de edad (media de 42.1 años). El $86 \%$ presentaba una patología dental de base (caries, gingivitis, periodontitis) y el $41 \%$ se había 
sometido a algún procedimiento dental (las extracciones dentales fueron el procedimiento más frecuente). Sin embargo, en este estudio no se menciona la cantidad de pacientes pediátricos incluidos, si los pacientes recibieron profilaxis antibiótica posterior a los procedimientos dentales ni si presentaban algún factor de riesgo para complicaciones ${ }^{6}$.

Se realizó una revisión sistemática para evaluar si el uso de antibióticos profilácticos en procedimientos e implantes dentales disminuía el riesgo de complicaciones infecciosas (bacteriemias e infecciones localizadas). Se obtuvieron siete estudios clínicos aleatorizados con un total de 1,368 pacientes a quienes se les realizaron extracciones o implantes dentales. No se encontró evidencia estadísticamente significativa que respaldara el uso sistemático de profilaxis antibiótica para reducir la falla de implantes $(p=0.09$; riesgo relativo [RR]: 0.43; intervalo de confianza del 95\% [IC95\%]: $0.16-0.14) \quad 0$ las complicaciones posoperatorias ( $p=0.47$; RR: 0.74 ; IC95\%: 0.34-1.65). Sin embargo, 33 pacientes recibieron antibiótico para prevenir una complicación ${ }^{15-17}$.

El uso de profilaxis antimicrobiana se recomienda en pacientes con riesgo de endocarditis (cardiopatías congénitas, reemplazo valvular, entre otros), inmunocomprometidos, aquellos con procedimientos extensos y prolongados, o con cirugía en sitios infectados e implantes de material ${ }^{16}$. De acuerdo con el National Institute for Health and Clinical Excellence (NICE), el cepillado dental presenta un mayor riesgo de endocarditis que un procedimiento dental, por la repetida exposición a bacteriemias de la biota oral17.

Los dentistas prescriben aproximadamente el $10 \%$ de todos los antibióticos. Los más utilizados como profilaxis para los patógenos comúnmente encontrados en la cavidad oral son amoxicilina, amoxicilina-ácido clavulánico, penicilina $\mathrm{V}$, cefalexina, metronidazol y clindamicina ${ }^{17}$.

En una revisión sistemática se investigó la eficacia de la amoxicilina y de la amoxicilina-ácido clavulánico en la reducción del riesgo de infecciones posoperatorias en cirugías del tercer molar. Se analizaron once estudios y ocho metaanálisis. Los resultados mostraron que el tratamiento con amoxicilina-ácido clavulánico reduce significativamente el riesgo de infección posterior a la extracción del tercer molar. Sin embargo, se debe utilizar con precaución, ya que en estudios clínicos en pacientes voluntarios sanos se observó un impacto negativo en la diversidad bacteriana y en la resistencia antibiótica ${ }^{18}$.

El abuso de antibióticos ha aumentado la resistencia microbiana y la alteración del microbioma, facilitando el excesivo crecimiento de levaduras y bacterias, como Clostridium difficile, y se ha provocado la interferencia con la absorción y el metabolismo de vitaminas y otros nutrientes, incrementando la susceptibilidad a futuras infecciones ${ }^{17,18}$.

La tríada característica de un absceso cerebral se presenta solo en el $20-30 \%$ de los casos: cefalea (60$70 \%$ ), fiebre (50\%) y focalización. Las alteraciones neurológicas dependen de la localización del absceso cerebral, y la región frontal es la más frecuente en los abscesos de origen odontogénico ${ }^{3,15}$. Por otro lado, la mayoría de los abscesos son únicos (81\%). Los estudios de elección para el diagnóstico son los de imagen. En el caso aquí reportado, en la TC y en la RM con gadolinio se observó una lesión hipodensa con realce capsular con el medio de contraste (Figura 2) ${ }^{11}$. La punción lumbar está contraindicada en la mayoría de los casos por el riesgo de herniación y de hipertensión endocraneana. Únicamente se logra aislar el agente etiológico en el $25 \%$ de los casos y los hemocultivos son positivos en el $28 \%$. El diagnóstico de elección es el cultivo del material drenado del absceso cerebral ${ }^{3}$.

Se requiere un manejo multidisciplinario con los servicios de terapia intensiva, neurocirugía e infectología pediátrica para la atención de estos casos. Es importante el inicio temprano de un tratamiento empírico dirigido a los microorganismos más comunes dependiendo del punto de entrada. En este paciente se utilizó una cefalosporina de tercera generación más metronidazol para cubrir los patógenos de la cavidad oral ${ }^{3}$.

El avance en las técnicas neuroquirúrgicas es una pieza clave en el pronóstico de esta patología. Actualmente, la aspiración guiada por estereotaxia es el procedimiento más utilizado; está indicada en los abscesos cerebrales mayores de $1 \mathrm{~cm}^{3,12}$. La resección total por craneotomía se realiza en pacientes con abscesos grandes, multilobulados, y con hipertensión endocraneana grave ${ }^{12}$. El adecuado manejo de las muestras del material drenado es importante para una identificación etiológica. Es necesario utilizar diferentes medios de cultivo en ambientes aerobio y anaerobio ${ }^{12}$.

E. corrodens es sensible a la penicilina, la ampicilina, las cefalosporinas de segunda y tercera generación, los carbapenémicos, las fluoroquinolonas y la trimetoprima-sulfametoxazol. En cambio, presenta resistencia a la clindamicina, la eritromicina y el metronidazol. En algunos casos se ha reportado la producción de betalactamasas, las cuales son inhibidas por el ácido clavulánico y el sulbactam ${ }^{9}$. En el caso de este reporte, a pesar del aislamiento de $E$. corrodens en el cultivo del material drenado, se continuó con un doble 
esquema de antibiótico, ya que existe una gran proporción de abscesos polimicrobianos ${ }^{12}$.

La duración del tratamiento de los abscesos cerebrales es variable, pues depende de la evolución clínica y radiológica de cada paciente. Se recomiendan tratamientos intravenosos por 4-6 semanas, valorando el cambio a la vía oral. Debido a la gran proporción de abscesos polimicrobianos ( $>30 \%$ ), se recomienda continuar con cobertura para anaerobios durante todo el tratamiento, aun con aislamiento ${ }^{12}$. Los estudios de imagen se deben realizar semanalmente para valorar la eficacia del tratamiento, y se debe observar la disminución del tamaño de la lesión, del edema y del efecto de masa. El tamaño del absceso disminuye en 1-4 semanas con terapia antibiótica o en combinación con aspiración por estereotaxia ${ }^{2}$. La resolución radiológica completa del absceso puede tardar 12-16 semanas, y el área de contraste residual puede continuar durante 6-9 meses posterior al tratamiento².

En un estudio multicéntrico retrospectivo de cuatro centros neuroquirúrgicos pediátricos en Inglaterra (1999 a 2009) se incluyeron niños de 1 a 17 años con diagnóstico de absceso cerebral. Se diagnosticaron 121 niños en un tiempo del diagnóstico de 7 días. Entre las manifestaciones clínicas más comunes se presentaron datos de focalización (53\%) y la tríada clásica de los abscesos en el $13 \%$. El $91 \%$ de los casos requirió tratamiento quirúrgico. Se obtuvieron cultivos positivos en el $85 \%$ : polimicrobianos (25\%), Streptococcus (50\%; más común del grupo milleri), anaerobios y bacterias gramnegativas (11\%). El tratamiento empírico combinado consistió en una cefalosporina de tercera generación y metronidazol por 6 semanas. Este estudio es el más grande que se ha realizado sobre abscesos cerebrales en niños en los últimos 20 años ${ }^{16}$.

El seguimiento de los pacientes pediátricos con abscesos cerebrales es semanal durante las primeras 2 semanas posteriores al egreso. Los estudios de imagen (RM o TC) se solicitan cada 2 semanas hasta que se presenta la resolución radiológica. Una vez resuelto, se deben realizar controles de imagen cada 3-4 meses durante 1 año, valorando si presentan recurrencias de la infección ${ }^{2}$. El éxito del tratamiento depende del paciente, pero sobre todo de la familia; se debe capacitar a los familiares sobre los posibles cambios neurológicos que podrían observarse y los datos de alarma. En caso de que el paciente presente fiebre, cambios en el estado de alerta, irritabilidad, disminución en la ingesta de alimentos, fontanela abombada, convulsiones o vómito en proyectil, los familiares deberán comunicarse con el médico tratante ${ }^{2}$.
El pronóstico en los niños depende de varios factores: la virulencia del patógeno, la localización y el número de abscesos, el origen de la infección y el estado clínico del paciente durante la presentación ${ }^{2}$. Se ha reportado que el $35 \%$ de los pacientes presentan un déficit neurológico a los 6 meses $^{16}$. Las secuelas neurológicas que con más frecuencia ocurren son epilepsia, déficit motor, alteraciones visuales y del aprendizaje, e hidrocefalia².

Los estudios sugieren una mortalidad de hasta el $33 \%$ para los abscesos cerebrales en niños. El estado neurológico del paciente al inicio de la presentación es un factor pronóstico; se ha observado una mayor mortalidad en aquellos que presentan un estado mental alterado y un rápido deterioro neurológico al ingreso. La mortalidad que se ha observado varía 2 : $0-21 \%$ en pacientes alertas, $60 \%$ en pacientes con signos de herniación y $89 \%$ en pacientes en estado de coma. La mortalidad sin tratamiento es del $20-50 \%$. Con un diagnóstico temprano, la recuperación es del $90 \%$,7,12,16.

En conclusión, los abscesos cerebrales de origen odontogénico y causados por $E$. corrodens en la edad pediátrica son de presentación muy escasa. En la actualidad, el uso de antibióticos profilácticos en procedimientos dentales es controversial, ya que están indicados en pacientes con factores de riesgo, pero se ha documentado que su uso indiscriminado aumenta la resistencia microbiana ${ }^{17}$. Aproximadamente uno de cada tres pacientes egresados con antibiótico no lo necesitaban ${ }^{17}$. Se recomienda que los clínicos consideren con precaución el uso apropiado de antibióticos para reducir la prescripción empírica (sin una identificación microbiológica) y dirigir un tratamiento adecuado. De esta forma se lograrán disminuir la presión antibiótica y la resistencia microbiana ${ }^{18}$. El avance en los estudios para el diagnóstico y el tratamiento multidisciplinario ha mejorado el pronóstico de los pacientes con abscesos cerebrales?

\section{Responsabilidades éticas}

Protección de personas y animales. Los autores declaran que para esta investigación no se han realizado experimentos en seres humanos ni en animales.

Confidencialidad de los datos. Los autores declaran que han seguido los protocolos de su centro de trabajo sobre la publicación de datos de pacientes.

\section{Derecho a la privacidad y consentimiento infor-}

mado. Los autores han obtenido el consentimiento informado de los pacientes o sujetos referidos en el artículo. Este documento obra en poder del autor de correspondencia. 


\section{Financiamiento}

Ninguno.

\section{Conflicto de intereses}

\section{Los autores declaran no tener ningún conflicto de intereses.}

\section{Bibliografía}

1. Pallesen LP, Schaefer J, Reuner $U$, Leonhardt $H$, Engellandt $K$, Schneider $\mathrm{H}$, et al. Multiple brain abscesses in an immunocompeten patient after undergoing professional tooth cleaning. J Am Dent Assoc. 2014;145:564-8

2. International Society for Pediatric Neurosurgery. Follow-up for brain abscesses in children. Ginebra: ISPN Guide to Pediatric Neurosurgery. Disponible en: https://www.ispn.guide/infections-of-the-nervous-system-in-children/brain-abscesses-in-children-homepage/management-of-brain-abscesses-in-children/follow-up-for-brain-abscesses-in-children/

3. Weinberg GA. Brain abscess. Pediatr Rev. 2018;39:270-2.

4. Karunakaran R, Marret MJ, Hassan H, Puthucheary SD. Eikenella corrodens from a brain abscess. Malays J Pathol. 2004;26:49-52.

5. Krzysztofiak A, Zangari P, De Luca M, Villani A. Brain abscesses: an overview in children. J Pediatr Infect Dis. 2019;14:2-5.

6. Moazzam AA, Rajagopal SM, Sedghizadeh PP, Zada G, Habibian M. Intracranial bacterial infections of oral origin. J Clin Neurosci. 2015;22:800-6.

7. Prasad KN, Mishra AM, Gupta D, Husain N, Mazhar H, Gupta RK Analysis of microbial etiology and mortality in patients with brain abscess. J Infect. 2006:53:221-7.
8. Gendron R, Greiner D, Maheu-Robert LF. The oral cavity as a reservoir of bacterial pathogens for focal infections. Microb Infect. 2000;2:897-906.

9. Jaramillo R, Suárez P, Barraza B, Lara P, Teherán L, Escamilla J. Eikenella corrodens: patogénesis y aspectos clínicos. Colomb Med. 2006; 37:228-41

10. Paul K, Patel SS. Eikenella corrodens infections in children and adolescents: case reports and review of the literature. Clin Infect Dis. 2001; 33:54-61.

11. Miranda HA, Castellar-Leones SM, Elzain MA, Moscote-Salazar LR. Brain abscess: current management. J Neurosci Rural Pract. 2013; 4:S67-81.

12. Sonneville R, Ruimy R, Benzonana N, Riffaud L, Carsin A, Tadié JM, et al. An update on bacterial brain abscess in immunocompetent patients. Clin Microbiol Infect. 2017;23:614-20.

13. Mueller AA, Saldamli B, Stübinger S, Walter C, Flückiger U, Merlo A, et al. Oral bacterial cultures in nontraumatic brain abscesses: results of a first-line study. Oral Surg Oral Med Oral Pathol Oral Radiol Endod. 2009;107:469-76.

14. Ewald C, Kuhn S, Kalff R. Pyogenic infections of the central nervous system secondary to dental affections - a report of six cases. Neurosurg Rev. 2006;29:163-6; discussion 166-7.

15. Asensi V, Álvarez M, Carton JA, Lago M, Maradona JA, Asensi JM, et al. Eikenella corrodens brain abscess after repeated periodontal manipulations cured with imipenem and neurosurgery. Infection. 2002;30:240-2.

16. Felsenstein $S$, Williams $B$, Shingadia $D$, Coxon $L$, Riordan $A$ Demetriades AK, et al. Clinical and microbiologic features guiding treatment recommendations for brain abscesses in children. Pediatr Infect Dis J. 2013;32:129-35

17. Gill AS, Morrissey H, Rahman A. A systematic review and meta-analysis evaluating antibiotic prophylaxis in dental implants and extraction procedures. Medicina (Kaunas). 2018;54:95.

18. Menon RK, Gopinath D, Li KY, Leung YY, Botelho MG. Does the use of amoxicillin/ amoxicillin-clavulanic acid in third molar surgery reduce the risk of postoperative infection? A systematic review with meta-analysis. Int J Oral Maxillofac Surg. 2018;48:263-73. 